\title{
CRISIS DE LOS CUIDADOS, LEY DE DEPENDENCIA Y CONFUSIÓN SEMÁNTICA
}

\author{
CARE CRISIS, DEPENDENCY ACT AND SEMANTIC MIX UP
}

\author{
Amparo Serrano Pascual aserranop@cps.ucm.es \\ Universidad Complutense de Madrid. España
}

Alba Artiaga Leiras alba.artiaga@egeco.es
Universidad Complutense de Madrid. España

María Celeste Dávila de León mcdavila@cps.ucm.es

Universidad Complutense de Madrid. España

\begin{abstract}
RESUMEN
La condición de dependencia, inmanente a todo ser humano, ha sido regulada política e institucionalmente en base a una serie de supuestos incuestionables que articulan una construcción sexualizada de los cuidados. Estos a priori podrían verse revisados con la implementación de iniciativas políticas como la Ley 39/2006, de 14 de diciembre, de Promoción de la Autonomía Personal y Atención a las personas en situación de Dependencia (Ley de Dependencia o LAAD) al poderse inducir una socialización de las obligaciones morales frente a la dependencia, una visibilización de las competencias y destrezas que reclaman los cuidados, y una revisión del "sexo" del trabajo. Este artículo discute, desde una perspectiva de género, el potencial herético de esta Ley y su contribución a una pugna por el sentido acerca de los cuidados. Para ello, este análisis crítico de la LAAD se apoya además en un trabajo de campo consistente en la realización de 20 entrevistas a informantes clave, con el que se trata de indagar en las luchas por el sentido entre diversos actores que participaron en la elaboración de la Ley.
\end{abstract}

Palabras Clave

Dependencia; Diversidad funcional; División sexual del trabajo; Ética de los cuidados; Organizaciones de mayores.

\begin{abstract}
The condition of dependence, inherent to all human being, has been regulated politically and institutionally on the basis of a series of assumptions which articulate a gender construction of care. These a priori's could be subject to an overhaul due to the implementation of political initiatives, such as the Dependency Act as it could induce to a socialization of moral duties with regard to dependency, a visibility of competences and skills required for care and a review of the "gender" of work. This article debates, from a gender perspective, the potential of this Act and its contribution to the fight for giving a meaning to care. This analysis is based on field work, consisting of 20 in-depth interviews with key persons, with the view to study the semantic battles between the various players which participated in the elaboration of the Act.
\end{abstract}

KEYWORDS

Dependency; Elder movement; Ethic of care; Gender division of labor; Impaired mobility. 


\section{INTRODUCCIÓN}

La creciente incorporación de las mujeres al mercado de trabajo, la prolongación de la esperanza de vida de la población y, con ello, la mayor presencia de situaciones de dependencia, la multiplicación de modelos familiares alternativos a la familia nuclear extensa, el proceso de europeización de la cuestión social, etc. han puesto en la agenda política la necesidad de revisar los acuerdos apriorísticos en los que se ha basado tradicionalmente el ejercicio de los cuidados. La condición de dependencia, inmanente a todo ser humano, ha sido regulada política e institucionalmente en base a una serie de supuestos incuestionables que explican la importante carga moral que ha disciplinado su ejercicio. Gran número de dimensiones que conformaban este "sentido común" acerca de los cuidados y de la atención a la dependencia, como son el espacio "natural" de su expresión (el ámbito doméstico y familiar), los sujetos de la acción de cuidar (mujeres), la naturaleza de su ejercicio (emocional), los criterios de su regulación (informalidad), 0 su dominio (privado) (Carrasquer 2013), pueden verse re-visados ${ }^{1}$ por esta confluencia de factores.

Esta situación ha suscitado un profundo interés que se ha traducido tanto en la multiplicación del número de estudios realizados, como en la orientación de políticas y dispositivos de intervención con los que hacer frente, desde diversas perspectivas, a la así llamada "crisis de los cuidados". Este creciente interés hacia el estudio, regulación y comprensión de los cuidados podría ser expresión, y a la vez catalizador, de importantes cambios en el orden social de género; de la "invención" de una nueva cuestión social que a modo de una nueva aporía permitiría a la sociedad cuestionarse a sí misma.

El objetivo de este artículo es indagar en los procesos de confusión semántica que está induciendo la crisis de los cuidados, y analizar el nuevo orden de clasificaciones y "di-visiones" (Bourdieu 1985) del orden social que se está promoviendo. Dicotomías de sentido que han articulado una doxa acerca del trabajo, del género y del orden político de la sociedad salarial, —como son las establecidas entre el espacio público y privado, emocional y racional, doméstico y productivo, o formal e informal- podrían verse reformuladas tras este desorden político y semántico que genera el cuestionamiento de los acuerdos políticos y sociales tradicionales bajo los que se desarrollan estas tareas. La Ley de Promoción de la Autonomía Personal y Atención a las Personas en Situación de Dependencia (LAAD) ha sido una de las respuestas/apuestas institucionales más importantes frente a esta situación de crisis de los cuidados.

El importante potencial de esta ley explica que haya sido objeto de numerosos estudios desde diversas perspectivas, que podríamos agrupar en tres bloques temáticos relacionados entre sí. En primer lugar, podríamos destacar aquellas investigaciones que

\footnotetext{
${ }^{1}$ Más que con los ojos, miramos a través de las palabras, y, por tanto, las luchas políticas son, también y sobre todo, batallas semánticas. Desde esta perspectiva, diversos movimientos sociales insisten en la necesidad de transformar los a prioris que articulan la noción de cuidados.
} 
valoran el impacto e idoneidad de la Ley de Dependencia desde un marco de análisis centrado en las políticas públicas y del bienestar, y en concreto, en la capacidad de dar cobertura a las necesidades de las personas en situación de dependencia. El centro de atención de estos estudios es la garantía de derechos sociales que proporciona a las personas mayores o con diversidad funcional, los modos de financiación o los procesos de mercantilización de la provisión de servicios (Casado 2004; Montserrat 2006; Rodríguez Cabrero 2007; entre otros). En este marco analítico, se ha estudiado, por ejemplo, la repercusión de la ley en la creación de empleo y en las nuevas necesidades formativas de los trabajadores (Herce, Labeaga, Sosvilla y Ortega 2006; Instituto Regional de las Cualificaciones de la Comunidad de Madrid 2010), análisis de los costes que supone o supondrá la ley (Dizy 2010), etc.

En segundo lugar, es particularmente notoria, desde hace varias décadas, la producción de estudios que, desde una perspectiva de género, investigan el grado en que las políticas sociales de los diversos regímenes de bienestar legitiman y/o promueven una organización social de los cuidados "familiarista". Estos estudios destacan la falta de atención prestada, por parte de los enfoques dominantes sobre políticas públicas, a la esfera familiar y privada, y subrayan la necesidad de introducir una perspectiva de género en la problematización de las cuestiones sociales a las que tratan de dar respuesta estas políticas. Esta perspectiva insiste en la necesidad de revisar el diseño de las mismas para integrar las vivencias invisibilizadas que experimentan muchas mujeres. Los cuidados aparecen definidos desde esta perspectiva como una dimensión central en el análisis del bienestar y de las políticas públicas (Fraser 1989; Orloff 1993; Sainsbury 1994; O'Connor 1996; Daly y Lewis 2000; Bettio, Simonazzi y Villa 2006; Arriba y Moreno 2009; Lewis 2011; entre otros). En España, contamos con un importante trabajo de evaluación de las políticas sociales y sus efectos en la redistribución de las responsabilidades sociales de los cuidados, principalmente centrado en el análisis de las políticas de conciliación y de igualdad (Carrasco, Borderías y Torns 2001; Torns 2007; Bustelo y Lombardo 2007; De Villota 2008; etc.).

Finalmente, podemos también destacar algunos estudios más específicos que, desde una perspectiva de género, analizan la LAAD como propuesta innovadora para la institucionalización de los cuidados (Pérez 2006; Rogero 2010; Peterson y Bustelo 2007; entre otros).

Una de las conclusiones fundamentales de estos estudios es el papel central que juegan las presunciones culturales, integradas en las intervenciones públicas, en la reproducción de relaciones políticamente asimétricas entre hombres y mujeres, y, en este sentido, la relación indisociable que se establece entre el significado cultural de los cuidados y la producción política de sujetos de género. Estas concepciones culturales hegemónicas son, en gran medida, el resultado de la movilización de actores sociales que han pugnado por hacer visible determinada concepción de la realidad. De hecho, otra importante conclusión de las evaluaciones y estudios realizados acerca de la Ley de Dependencia incide en señalar el papel central que juegan las coaliciones de actores e instituciones y las formas de movilización que se han articulado en torno a la ley en 
la forma específica que ha adoptado ésta y, por tanto, en sus consecuencias (véase Rodríguez Cabrero 2011; Marbán 2012). En este sentido, diferentes autores (Bustelo y Lombardo 2006; SIPOSO 2008; Martín Palomo 2010; Díaz Velázquez 2010; entre otros) han alertado del importante papel que juegan los marcos conceptuales con los que se designa la ley para entender el alcance de esta. A pesar de ello, son escasos los estudios que evalúan y analizan la ley desde esta perspectiva. Menos habituales son también las investigaciones que plantean el estudio de la hegemonía de unos a prioris culturales en función del proceso de producción política de esta ley, y el papel central de los actores colectivos que participaron en su elaboración. La hegemonía de determinadas "voces" para modificar el ámbito de lo nombrable es el resultado de un frágil equilibrio de fuerzas asimétricas entre estos actores. Los conceptos de dependencia y de cuidados no sólo nombran la situación (ubican una denominación en un marco de sentido), sino que pueden servir como recursos estratégicos en manos de diversos movimientos sociales para impulsar sus propuestas en la agenda política.

Este trabajo intenta participar en este debate de evaluación de la LAAD a partir del análisis de la contribución de la ley a esta pugna por el sentido, y, de este modo, vamos a prestar una particular atención a las preconcepciones (conjunto de creencias y conocimientos) acerca de los cuidados que moviliza la ley. Trataremos así de indagar en la participación de esta ley en la producción política de los sujetos de los cuidados y del verbo cuidar. El signo (en este caso, nociones como "cuidados"), tal y como plantea Bajtin (1979), es un espacio esencial en donde se enfrentan series diferenciadas y, a veces contrapuestas, de campos isotópicos y, por tanto, permite anclar distintos puntos de vista. Por ello, el estudio crítico de la Ley de Dependencia no puede desvincularse del análisis de los discursos de personas que representan a grupos sociales que han pugnado por hacer prevalecer un modo específico de entender qué significa la dependencia, los cuidados y qué distribución de las responsabilidades aparece como la más justa y adecuada para hacer frente a esta crisis del "cuidar".

Este estudio se ha basado, por tanto, en el análisis crítico de la Ley 39/2006 (LAAD) y en el análisis de los discursos de 20 informantes clave. El trabajo de campo fue realizado en el marco de los proyectos FEM2010-18827, financiado por el Ministerio de Ciencia e Innovación, y 4164391 - 8/10, financiado por el Instituto de la Mujer. El principal criterio de selección para el diseño de la muestra de estos informantes clave ha sido el criterio de diversidad y peso de posiciones y perspectivas (voces) de diversos actores que participaron directa e indirectamente en el diseño de la ley. Tal y como se argumentó en un trabajo anterior (Artiaga Leiras y Serrano Pascual 2012), para entender el diseño específico de la ley es importante considerar los diversos procedimientos de intervención política en manos de los actores que han participado en su constitución: los ritos de institución y tecnologías de legitimación promovidos como antesala de la ley, uno de cuyos mejores exponentes es el Libro Blanco de la dependencia; los espacios de participación previstos institucionalmente, como es el diálogo social entre los interlocutores sociales y actores gubernamentales, los Consejos Estatales y Órganos Consultivos tripartitos, los Foros de participación política, el Parlamento; y las vías 
más informales que han promovido un orden deliberativo, como son las plataformas de movilización, las protestas, las iniciativas de algunas personas². En este sentido, y a fin de asegurar la diversidad de posiciones que expresa esta heterogeneidad de actores que participaron en la elaboración de la ley, hemos seleccionado una muestra que obedece a tres principales criterios: la entidad a la que pertenecen, la vinculación con la ley, y el tipo de responsabilidad e implicación en la elaboración de esta. En base a estos tres criterios, hemos realizado entrevistas a diversos informantes clave: expertos que participaron en la elaboración del Libro Blanco; expertos en cuestiones de género; representantes del Instituto de Mayores y Servicios Sociales, (Instituto de Mayores y Servicios Sociales, IMSERSO) y otras organizaciones de mayores (Unión Democrática de Pensionistas, UDP); representantes de movimientos sociales de mujeres (Asamblea Feminista de Madrid, Forum de Política Feminista) y de instituciones representativas de sus voces (Instituto de la Mujer); representantes de organizaciones de mujeres mayores (Asociaciones de Mujeres por un Envejecimiento Saludable, AMES); representantes de las asociaciones patronales de servicios a la dependencia (Fundación ALARES; Asociación de Empresas de Servicios para la Dependencia, AESTE); representantes de departamentos de políticas sociales de asociaciones sindicales (Comisiones Obreras, CCOO); representantes de organizaciones de personas con discapacidad/diversidad funcional (Comité Español de Representantes de Personas con Discapacidad y el Foro de Vida Independiente, CERMI); representantes de trabajadores de los servicios sociales, responsables de cuestiones vinculadas con la dependencia (seguimiento, asesoría, programas dirigidos a cuidadores, etc.)

Para llevar a cabo el análisis de la LAAD, nos hemos basado fundamentalmente en la perspectiva de análisis crítico de discurso, particularmente aquella elaborada por autores como Weiss y Wodak (2008) o Fairclough (1995), enfoque éste que mantiene una larga tradición en el análisis de la legislación. Hemos prestado una particular atención a aquellos a priori que plantea la ley (y las pugnas por el sentido), así como los recursos retóricos con los que se construye un sentido común acerca de los cuidados, esto es, un modo de entender qué significa la dependencia, el cuidar, y quién es el sujeto natural de los cuidados.

Esta reflexión se articula en dos fases. En un primer momento, se plantea un breve análisis del potencial herético de la ley, dirigido fundamentalmente a estudiar qué cambios epistémicos en torno a la cuestión de los cuidados podría inducir la LAAD (1). En un segundo momento, nos orientamos a identificar los marcos de comprensión de los cuidados que plantea la ley (concepción de dependencia, del sujeto de cuidados, y de qué significa cuidar) y su relación con las voces de diversos actores que, individual y/o colectivamente, han participado en su definición (2). Concluimos este artículo con algu-

\footnotetext{
${ }^{2}$ Por problemas de espacio, no podemos desarrollar más exhaustivamente el estudio de la genealogía de la ley y de los discursos y posiciones de los actores que participaron en esta; sin embargo, remitimos al lector a Rodríguez Cabrero (2007) y Marbán (2012).
} 
nas breves reflexiones críticas sobre el alcance de esta medida con un gran potencial emancipador, no sólo por su capacidad de responder a la condición de vulnerabilidad de gran número de sujetos en situación de dependencia, sino también de "desordenar" semánticamente las asunciones de género que conforman las relaciones políticas asimétricas entre los sexos.

\section{Potencial "herético" de LA LAAD. Un ANÁLISIS DESDE UNA PERSPECTIVA DE GÉNERO}

La LAAD supuso un avance importante en la politización de una cuestión, connatural en el ser humano, como es la interdependencia, que, en determinadas fases de la vida de la persona, se expresa de forma más ineludible (Martín Palomo 2010). Por su capacidad potencial de replantear algunas de las cuestiones nucleares que han regulado la construcción sexuada de los cuidados, esta ley podría estimular una nueva aporía e inducir la capacidad de una sociedad de pensarse a sí misma. De las muchas que podríamos plantear, tres cuestiones nos parecen particularmente importantes por su carácter transgresor, esto es, susceptibles de "someter a disputa" nociones que se daban por supuesto y que contribuían a reproducir el orden político vinculado al género:

a) una posible desfamiliarización y replanteamiento político de la distribución de las responsabilidades sociales frente a los cuidados entre los diversos actores sociales (familia, individuo, estado, mercado, etc.) y del papel de la familia (mujeres) como único factor de solidaridad y de bienestar social;

b) la capacidad de poder revalorizar actividades que, a pesar de su complejidad e intensidad, apenas han sido visibles y/o reconocidas socialmente;

c) y una reinvención del (sexo del) trabajo (Hirata y Zariffia 2007) (y las di-visiones semánticas planteadas entre el trabajo productivo y el doméstico) tras el cuestionamiento de su contenido simbólico.

En primer lugar, la LAAD puede inducir una politización de la dependencia vinculada al envejecimiento o/y diversidad funcional y, con esta, una socialización de las obligaciones morales (Rodríguez Cabrero 2007). La satisfacción de las necesidades que plantean estas situaciones de dependencia deja de estar regulada únicamente por el contrato moral que toda mujer contrae con la sociedad, y se promueve un modo de discutir y pensar esta situación en términos colectivos y políticos.

La configuración de la dependencia como objeto de específica regulación jurídica implica un cambio de perspectiva. Conduce a la definición de nuevas funciones que debe cumplir el Estado. Aún a pesar de los tímidos recursos que esta ley ha puesto a disposición de la sociedad para su implementación, la mera redacción de una ley como esta podría inducir una posible revisión de un acto de adhesión dóxica. Las demandas sociales que se promueven tras la acentuación de la moral del "sujeto activo" en todo el colectivo social, la mayor presencia política de los colectivos de mayores y de diversidad 
funcional, la europeización de la cuestión social, las condiciones de precarización laboral que, junto con la conciencia política de la importancia de la autonomía económica, propician la incorporación de las mujeres en el mercado de trabajo, etc. pueden adoptar una naturaleza contradictoria. Estas contradicciones sociales están siendo interpretadas y vivenciadas por parte de la población como ambivalencias personales que deben ser resueltas en el seno de la familia a través de las estrategias asi llamadas de "conciliación $^{3 \prime}$. Este discurso individualizador y potencialmente responsabilizante de la conciliación puede verse impugnado con la proclamación de iniciativas como la LAAD. En la redacción de la ley, se visibiliza el papel del Estado (como representación de lo colectivo) en la búsqueda de una solución a esta crisis de los cuidados, lo que podría inducir un cambio en su representación como un dilema familiar (y personal de las mujeres).

Otra cuestión importante que puede promover la ley es la revisión de los criterios de valoración de las actividades vinculadas a los cuidados que, al ser posible objeto de una remuneración, no sólo permite hacer más explícito el carácter contractual (frente al natural) de ejercicio de estas tareas, y, por tanto, intrínsecamente voluntario, sino que también posibilita traducir estas actividades a un lenguaje visibilizador que otorga valor, su equivalente monetario en el mercado. Podría así permitir valorar habilidades diferentes a las asociadas al imaginario masculino, como es la inteligencia emocional, pudiendo así crear nuevos modos de clasificación social (Illouz 2010). Esta ley visibiliza la necesidad de recursos y, por ello, tanto si la respuesta se busca en el mercado como en los servicios públicos o la familia, esta situación requiere una inversión social. Esto está produciendo un curioso proceso político, que se ha traducido en un número importante de iniciativas tanto de los poderes públicos como de actores privados, patronal y sindicatos, acerca de la necesidad de profesionalizar los cuidados. Independientemente del carácter desvalorizador de los cuidados informales que plantea este discurso acerca de la profesionalización y catalogación de los cuidados, este movimiento hacia la profesionalización de los cuidados está también promoviendo su visibilización y reconocimiento. Un ejemplo es el proceso de acreditación de las competencias vinculadas al ejercicio de los cuidados (Real Decreto 1379/2008, en donde se definen los Certificados de Profesionalidad). La "invención" de los cuidados como categoría profesional ha fomentado un ritual dirigido a la observación minuciosa de las destrezas que estas tareas generan, su descomposición en habilidades, facilitando su codificación y regulación. La activación de este ritual de institución permite asignar esencias y propiedades a determinados tipos de actividades, y posibilita el reconocimiento de un rito de paso por el que las actividades entran en un nuevo campo semántico. La certificación permite así la afirmación ritual de una delimitación entre dos modos de percibir y asignar significado y valor a las activida-

${ }^{3}$ La noción de conciliación presupone la presencia previa de un conflicto en el que el sujeto participa y tendría que resolver. Esto explica que otros espacios semánticos en los que se usa esta noción sean el derecho (asociado a un contrato voluntario), la religión (tras el perdón otorgado por Dios tras una infracción moral), y el ámbito de la familia. 
des. En un momento en el que sólo lo que es posible traducir en números es objeto de valor, este proceso de cuantificación de las destrezas vinculadas a los cuidados puede tener importantes repercusiones en su visibilización y valoración. Es más, la presencia de un certificado que acredita socialmente la disposición de una serie de competencias que cualifican para el ejercicio de los cuidados, cuestiona una representación esencializante según la cual el ejercicio de estas tareas reclamaría la movilización de destrezas naturalmente (biológicamente) concedidas a las mujeres.

Una tercera dimensión de la ley que podría impugnar algunos de los principios que articulan el contrato entre los géneros es la mercantilización de los cuidados. El hecho de recibir una subvención o paga por el ejercicio de las tareas vinculadas a la dependencia podría poner en cuestión la dicotomía jerárquica semántica y política establecida entre el trabajo (como actividad inserta en el mercado) y los cuidados (vinculada a los afectos). En torno a esta dicotomía se articulan los criterios de dignificación y de atribución de poder entre los sexos.

Sin poder entrar en detalle en otras cuestiones que suscita la ley, análisis que ya han sido emprendidos en interesantes trabajos anteriores (Rodríguez Cabrero 2007; 2010; 2011; Rogero 2010; Marbán 2012; etc.), queríamos centrarnos más bien en el modo de entender la dependencia, el sujeto de cuidados y el contenido legítimo de la actividad de cuidar, concepciones estas que, desde nuestro punto de vista, están restringiendo, en gran medida, este potencial emancipador y transgresor que podría ejercer la ley.

\section{LAS PUGNAS POR EL SENTIDO. REPRESENTACIONES Y VOCES HEGEMÓNICAS EN LA LAAD}

En torno a la noción de dependencia y de cuidados se van a movilizar gran número de actores que ven en esta definición no sólo los espacios legítimos de intervención, sino también una definición del orden normativo que organiza las responsabilidades y su distribución entre los diversos actores sociales.

\section{Las doxas que induce la ley: la noción de dependencia}

En primer lugar, esta ley plantea una representación principalmente física de la dependencia, que evoca un marco gerontológico y médico (Díaz Velázquez 2010), y que, por tanto, reclama como espacio natural de expresión, el ámbito privado e íntimo del hogar (o centros que reproducen las condiciones del hogar).

"Se entiende como dependencia el estado de carácter permanente en que se encuen-
tran las personas que, por razones derivadas de la edad, la enfermedad o la disca-
pacidad, y ligadas a la falta o a la pérdida de autonomía física, mental, intelectual o
sensorial, precisan de la atención de otra u otras personas o ayudas importantes para
realizar actividades básicas de la vida diaria o, en el caso de las personas con discapa- 
cidad intelectual o enfermedad mental, de otros apoyos para su autonomía personal" Autonomía: "capacidad de controlar, afrontar y tomar, por propia iniciativa, decisiones personales acerca de cómo vivir de acuerdo con las normas y preferencias propias así como de desarrollar las actividades básicas de la vida diaria..." (LAAD, art. 2, BOE, 14.12.2006)

La atención será así entendida en términos médico-sanitarios, y dentro de este modelo médico (paradigma rehabilitador ${ }^{4}$ ), se prioriza la rehabilitación funcional (Díaz Velázquez 2010). La satisfacción de estas necesidades es entendida como un ejercicio de promoción del autoabastecimiento. En este sentido, es una representación privada que hace al espacio familiar (el hogar) el ámbito lógico y natural para su desempeño.

Esta representación de la dependencia se vincula fundamentalmente con el cuidado del cuerpo (y de sus necesidades) y refuerza una concepción que hace a la familia y al "hogar" el espacio más adecuado para una intervención que reclama traspasar el dominio custodiado de la intimidad (etimológicamente, el superlativo de "interior"). El espacio íntimo es construido en nuestras sociedades como un ámbito de preservación del sujeto frente a la sociedad, allí donde lo público (entendido como lo ajeno) no puede penetrar. Es un área protegida de la intromisión de la mirada ajena (espacio desligado de la existencia pública) a través de diversos mecanismos de regulación, unos de carácter más político, como es la garantía mediante leyes que preservan el derecho a la protección contra la invasión a la intimidad y a la privacidad, y otros de naturaleza más moral, como es la inducción del sentimiento de pudor, entendido como salvaguarda y orden regulado de las miradas. La invasión de este espacio puede ser por tanto vivenciado como un ejercicio de violencia y de humillación. Estos mecanismos sociales de regulación del espacio íntimo contribuyen a la familiarización de los cuidados, ya que los miembros de la misma familia (cercanos a este espacio socialmente construido como "interior") presentan mayor "autoridad" para traspasar este espacio de privacidad e intimidad. Esta ley desarrolla, por tanto, una perspectiva individualizadora en donde el espacio de autonomía a promover sigue siendo el de la esfera privada e intima, desvinculada de la pública.

Sin embargo, y por otro lado, esta representación médica de los cuidados podría facilitar una revisión de la institucionalización de los espacios de silencio político al que hemos abocado a los cuerpos, al promoverse una representación de estos como espacio de intervención médica de profesionales ajenos al hogar. Podría también hacer más visible y pública la vulnerabilidad que caracteriza a todo sujeto, vinculado a un cuerpo silenciado, pero que cuando se expresa de forma ineludible, manifiesta la naturaleza del cuerpo doliente y dependiente (Nancy 2006). Esta concepción podría facilitar el cues-

\footnotetext{
${ }^{4}$ Aunque la lógica es médica, sin embargo, tal y como señala Rodríguez Cabrero (2007), la posición del sistema sanitario, por razones muy diversas (necesidad de marcar diferencias entre identidades profesionales, evitar la extensión de la lógica sanitaria de la gratuidad en los servicios sociales, etc.), es definida en la ley de forma débil e imprecisa.
} 
tionamiento de las representaciones del sujeto como un ente acabado, cuyo cuerpo desaparece, y sólo se manifiesta, de forma patente, cuando este se fractura (dolor, fatiga, lesiones, etc.). Este cuerpo dependiente es un cuerpo que habla, que cuestiona esta visión holista y plantea la naturaleza vulnerable del sujeto y su carácter abierto e inacabado (e interdependiente). Esta intervención en el cuerpo, a través de la proliferación de trasplantes, de implantes, sometido a una disección ingenieril, sitúa a estos cuerpos en una permanente exposición pública (Nancy 2006). El cuerpo pasa así de ser un contenedor a estar permanentemente reconstruido e intervenido. Esta intimidad expuesta permite mostrar el carácter indefinido del cuerpo, en donde está presente la ajenidad (Nancy 2006). Esta redefinición del cuerpo podría servir para cuestionar el referente ontológico de la modernidad, que hace al individuo un ser autónomo, cerrado y autocontenido. Sin embargo, este cuerpo que habla es también relegado al silencio, a través de la movilización de mecanismos de interacción de diversa índole, como es la infantilización, o la despersonalización de las personas expuestas, la producción política de entidades incompletas, o la imposición de cortinas que limitan la exposición de los cuerpos.

El ejercicio de autonomía, entendido como el gobierno de las actividades básicas de la propia persona, es construido como una condición de dignificación y de legitimación pública. El uso permanente en la ley de términos como "capacidad de controlar", "afrontar", "propia iniciativa", "decisiones personales" permite activar un marco individualizador que hace de la autonomía no sólo un objetivo sino también un deber moral de todo sujeto que se precie como tal. La autonomía, entendida como independencia y posesión de recursos materiales, deviene un modelo de normalidad, obviando la naturaleza vulnerable que caracteriza a todo ser humano (Martín Palomo 2010).

En la noción gerontológica y médica que se mantiene en la ley de lo que significa la dependencia, estructurada por niveles o estatus de pérdida de autonomía física, ha jugado un papel fundamental la definición de partida que ha planteado el IMSERSO ${ }^{5}$, en ese tándem con el que se ha asociado al discurso de la Unión Europea (UE) ${ }^{6}$. Por ello, la hegemonía de esta concepción médica puede ser el resultado de una dependencia

${ }^{5}$ Tres características definen los modos de negociar autoridad por parte de los representantes del IMSERSO, particularmente importantes en la redacción del Libro Blanco, la apelación a la participación de diversos expertos, muchos de ellos vinculados al IMSERSO, la presentación como un dia-logo(s) entre diversos actores y la evocación del "master symbol" (Turner 1997) en el que se ha convertido la UE.

6 "Se tomó de la Unión Europea. La definición de dependencia aparece en la ley. Lo que ocurre es que en principio la idea era meter sólo a personas mayores, o de ahí surgió. Es decir, cuando se ha hablado de dependencia, es decir, hoy ya nadie cuestiona qué es dependencia en España, pero entonces, cuando se hablaba de ley de dependencia estaba encuadrada y contextualizada en personas mayores" (Representante 2 del IMSERSO).

"Las personas que tienen dependencia son aquellas que no son capaces de hacer las actividades básicas de la vida diaria y necesitan a una persona para hacer las actividades básicas de la vida diaria. Estamos hablando de personas que no pueden comer, no pueden beber, no pueden asearse, no son capaces de orientarse. Esas son las personas que atendemos" (Representante 3 del IMSERSO). 
de la senda institucional (path dependency), y concretamente de las inercias planteadas por intervenciones anteriores de las que la LAAD es heredera ${ }^{7}$ (Rodríguez Cabrero 2011; Marbán 2012), pero también ha jugado un papel central la importante participación en la elaboración de la ley de las voces de actores como el IMSERSO y otros colectivos de mayores.

La dependencia pasa así a vincularse a la ausencia de salud que incapacita la autonomía para satisfacer sus necesidades básicas y, por tanto, es asociada a asistencia socio-sanitaria, siendo la categoría básica referencial de este programa la así llamada "tercera edad". Esta concepción hegemónica explica que las competencias administrativas en la aplicación de la ley a nivel estatal hayan sido transferidas del Ministerio de Trabajo al de Sanidad.

La definición de partida de la dependencia se caracteriza, por tanto, por tres rasgos: su naturaleza unidimensional (pérdida de autonomía física o intelectual), su emplazamiento semántico en el espacio de la privacidad e intimidad (y, por tanto, desmembrado del espacio político) y su carácter escalar (graduación de niveles o estatus), que orienta una representación individualizadora y despolitizante de la dependencia. Este estado es construido de forma administrativa (y parcialmente médica, en función de un dictamen clínico), a partir del desarrollo de instituciones destinadas a medir y controlar la dependencia. Así, la dependencia va a ser objeto de un pormenorizado recuento estadístico y de una detallada clasificación de sus diversos niveles y grados. La distancia a una norma de autonomía va a configurar los nuevos criterios de clasificación de los demandantes de ayuda (capítulo III del título I de la LAAD).

Esta noción de dependencia podría suscitar el cuestionamiento del referente epistemológico de la modernidad (el yo autosuficiente), que hace a los sujetos propietarios de sus cuerpos y plantea una visión ambivalente, como es la que se mantiene en el derecho moderno acerca de la responsabilidad, entre ser autor y ser actor del comportamiento. García (2011) plantea que, si bien el propietario de las acciones es aquel que las autoriza, el establecimiento de criterios de cuantificación de la "propiedad" corporal podría inducir una fractura en la constitución del yo y una revisión de la trascendencia del ego. Sin embargo, su relegación a un ámbito de recomposición reparadora (la rehabilitación del yo) impide esta impugnación política del sujeto, circunscribiéndolo al ámbito de la intervención médico rehabilitadora.

El concepto de dependencia que se propone es, por tanto, una noción cuantificable, en la que se establecen niveles o estatus (dependencia moderada, severa y gran dependencia) articulados jerárquicamente según su distancia a la norma de autonomía.

\footnotetext{
7 “En España, el marco de referencia era el plan gerontológico que tenía pocos años de vigencia del 91; y en el 96, se aprueba el Plan de Acción para personas con discapacidad, con unos marcos de referencia más genéricos, pero que ya de alguna manera se contemplaba el tema de la dependencia. En este contexto, empieza a trabajarse en el IMSERSO en estructurar un nuevo marco normativo específico en materia de dependencia" (Representante 1 del IMSERSO).
} 
Estos estatus son objeto de una minuciosa medición y regulación estadística, contribuyéndose así a la producción de nuevas categorías estadísticas según la distancia a la autonomía física. Cada estatus es reducido a un grado suficiente de homogeneidad para ser codificado bajo indicadores estadísticos que miden la capacidad de funcionamiento autónomo (capacidad de comer y beber, regulación de la micción y de la auto-higiene, desplazamiento autónomo, etc.). Esta percepción cuantitativista de lo que significa la dependencia puede haberse agudizado por la ausencia de recursos económicos e institucionales con los que se ha acompañado a la ley, que, al traducirse en una jerarquía de prioridades, permite restringir el acceso administrativo a algunas personas en situación de dependencia. De este modo, la ley aparece como activa aunque sólo un sector, calificado como de gran dependencia, pueda acceder a esta intervención social. Esta situación se ve agudizada tras los recortes propuestos recientemente (Moratoria del Consejo de Ministros en 2012) ${ }^{8}$. Esta acepción médico gerontológica hegemónica que induce una visión individualizadora y privatizante de lo que significa la dependencia es contestada por otros grupos sociales, que han hecho de la lucha por asignar un significado a los conceptos uno de los frentes más importantes de orientación de estas batallas políticas. Un ejemplo interesante es el Foro de Vida Independiente, que ha pugnado por hacer prevalecer una noción más pluridimensional y política de lo que significa la dependencia y autonomía. Esto supondría poner el acento no tanto en una intervención médica rehabilitadora como en una mediación política (por ejemplo, ordenamiento urbano) que garantice el ejercicio a estas personas de sus derechos de formación, ocio, etc. Según esta representación, la autonomía no se restringe a sus componentes puramente físicos y clínicos, sino también a la participación social y política y, por tanto, reclama una respuesta mucho más global y extrafamiliar que la propuesta en la ley. El espacio natural para la intervención, desde esta perspectiva, no sería el ámbito privado y familiar, sino público y político.

"Mi definición política: Soy una persona que funciona de otra manera... Entonces, cuando rompes ese concepto todo es... es como si te cambiaras de gafas. (...) Igual que tú no te defines por lo que no puedes hacer, yo tampoco. (...) Y lo del repago, vosotros lo llamáis copago pero eso no es así, es decir, yo ya pago mis impuestos pero además tengo que pagar por ser diferente" (Representante Foro de Vida Independiente).

Conscientes del papel central que desempeñan los a priori en la orientación de la mirada que se dirige al problema, estos actores reclaman un cambio conceptual como requisito nuclear para inducir una transformación política. Particularmente significativo de este movimiento social es su pugna por el significado con el que vincular la dependen-

\footnotetext{
${ }^{8}$ Esta moratoria reformula el concepto y los grados beneficiarios al reducirse las cuantías presupuestarias, lo que implica que el $26,3 \%$ de las personas con algún grado de dependencia, es decir, 398.519 personas, cuyo caso ya ha sido dictaminado, van a ser objeto de recortes presupuestarios (SAAD-IMSERSO 2012).
} 
cia, y su énfasis en transformar la denominación de autonomía (de autonomía funcional a diversidad funcional). Se plantea la necesidad de revisar las responsabilizaciones que van vinculadas a determinadas representaciones de la realidad y pasar de una concepción individualizante (problema de salud) a otra más política (una cuestión de derechos) (Guzmán, Moscoso y Toboso 2010). La cuestión sometida a problema no es la relación de dependencia con la sociedad, sino las relaciones asimétricas de poder establecidas, que contribuyen a reproducir un estatus de "normalidad".

Otras voces que han cuestionando esta visión individualizadora de la dependencia son las de los movimientos feministas vinculados en gran medida al mundo académico, que inciden en la necesidad de reformular las representaciones epistémicas y ontológicas que subyacen en este tipo de debates, y que hacen del sujeto mónadas autónomas y entidades aisladas.

"El asistente personal. Es lo único que hablan, de la autonomía en toda la Ley ¿sabes? Entonces, nos parecía que era algo, que bueno, que era una cosa muy puntual, pero que no extendía esa perspectiva de la autonomía personal al resto. (...) O sea, se hablaba solo... La Ley va dirigida a una población en concreto ¿no? Que son las personas mayores, dependientes y enfermas (Representante de la Asamblea Feminista de Madrid).

No, mire usted, yo quiero seguir siendo una persona más que tiene más años, nada más. Es lo único que tengo de diferencia con los demás. Pero sigo teniendo mi vida y mi vida activa. Quiero envejecer activamente" (Representante de la AMES).

Estas voces batallan por hacer de la dependencia no una condición excepcional, sino inmanente a cualquier sujeto. La vulnerabilidad hace de la interdependencia un rasgo constitutivo de la condición humana. Estas voces reclaman el establecimiento de mecanismos que permitan visibilizar la interdependencia, y facilitar así la politización y desfamiliarización en el encuadre del debate.

\section{La representación del sujeto de cuidados}

En segundo lugar, se puede destacar el sujeto de cuidados que se asume en la ley. De la reflexión anterior se concluye que el sujeto principal y "natural" para la satisfacción de estas necesidades es la familia. El papel de la mujer que subyace en esta noción familiarista se invisibiliza y se desplaza metonímicamente por la noción de "familia". La mujer aparece como sinécdoque, representación y esencia de lo familiar. Con esta operación retórica, de carácter metonímico, se recontextualizan los cuidados en el espacio privado, femenino, familiar. De hecho, tres de cada cuatro cuidadores principales son mujeres, en gran medida familiares de la persona dependiente (Observatorio de la Discapacidad 2008) ${ }^{9}$.

\footnotetext{
${ }^{9}$ Según el IMSERSO, en noviembre del 2012, el 44,73\% del total de las ayudas estaba vinculado a la prestación económica de cuidados familiares frente a otras prestaciones como la atención residencial, que
} 
Esto explica que, a pesar del carácter excepcional bajo el que se presenta en la ley la atención a la dependencia en el contexto familiar, la opción escogida haya sido, en la mayoría de los casos, los cuidados familiares.

Este juego de equivalentes semánticos, por el cual lo familiar se asocia con lo femenino, emocional e informal, se manifiesta en su particular definición de los perfiles de cuidadores. Todo ejercicio de clasificación induce un orden político. En el artículo 2 del título preliminar de la ley, se diferencia entre cuatro perfiles de cuidadores. Un primer perfil, que denominan "cuidadores no profesionales", presenta como característica distintiva y criterio clasificador el espacio social en el que se desarrollan las actividades de cuidados: la familia.

"Se entiende como 'cuidados no profesionales': la atención prestada a personas en situación de dependencia en su domicilio, por personas de la familia o de su entorno, no vinculadas a un servicio de atención profesionalizada" (LAAD, art. 2)

Un segundo perfil serían los cuidadores profesionales, que aunque no es incompatible con el cuidado familiar, sin embargo, se presenta como su álter ego ${ }^{10}$. El criterio de clasificación es, en este caso, el estatus social de la tarea. Un tercer perfil sería el asistente personal. El criterio en este caso no es el carácter profesional o el espacio de ejercicio de la tarea, sino los objetivos que persigue. Esta figura responde a las demandas del colectivo de sujetos con diversidad funcional, y configura un ethos específico de entender los cuidados, tal y como hemos desarrollado anteriormente. Finalmente, se expone un cuarto perfil de cuidadores, que corresponde con el tercer sector, cuyo criterio distintivo sería la naturaleza de la regulación de estas actividades ("responder a criterios de solidaridad, con fines de interés general y ausencia de ánimo de lucro", LAAD, artículo 2) y, en este sentido, los fines que persigue. Se establece así la distinción entre aquellas actividades con un carácter voluntario y solidario y aquellas que persiguen fines lucrativos.

Como detrás de toda operación de clasificación hay una división política, es fundamental analizar los criterios que justifican esta clasificación. En esta diferenciación de perfiles se mezclan un conjunto de criterios, la familiarización o no, cuyo principio explícito sería los mecanismos de regulación (profesionales, morales, etc.), el modelo de cuidados que plantea, o los fines que persiguen. Además del carácter político de estos criterios de delimitación y clasificación de las personas que cuidan (¿por qué las actividades realizadas por una ONG son conceptualizadas bajo el marco semántico de la voluntariedad y de la solidaridad y no lo son las actividades realizadas por las mujeres en el seno de las familias?), podría también objetarse su carácter naturalizador, al

representa tan sólo el $13,08 \%$ del total, la teleasistencia (13,59\%) o la ayuda a domicilio (12,87\%).

10 "Cuidados profesionales: los prestados por una institución pública o entidad, con o sin ánimo de lucro, o profesional autónomo entre cuyas finalidades se encuentre la prestación de servicios a personas en situación de dependencia, ya sean en su hogar o en un centro" (LAAD, artículo 2). 
plantearse una jerarquía entre las actividades de cuidados, situando en la escala más baja al espacio de cuidados vinculado con lo femenino (cuidados realizados en el ámbito doméstico de forma informal y aparentemente voluntaria por las mujeres).

No sólo se encuentra ausente en el texto de la ley un marco interpretativo en términos de género, sino que también contribuye a reproducir las asunciones sociales en las que se fundamenta el género como jerarquía social. No es, por tanto, la actividad de cuidados la que resulta invisible, sino sus condiciones de desarrollo. Sólo cuando esta se realiza en el espacio semántico vinculado con lo "femenino" (con su cadena de equivalentes: familiar, voluntario, informal), los cuidados son invisibilizados. Esta invisibilización implica no sólo que no se problematice el modo de organizar el ejercicio de estas actividades, sino también la escasa conciencia social y política de la presencia de una serie de necesidades, y la complejidad y la densidad de las actividades que conlleva su satisfacción.

Otra cuestión que refuerza el papel que se asigna a la mujer como sujeto de los cuidados es el hecho de que, si bien la ley plantea una multiplicidad de opciones para llevar a cabo estas actividades de promoción de la autonomía, la única de las fórmulas exentas de copago es la prestación económica en el entorno familiar. De este modo, no sólo se está perfilando, de nuevo, una jerarquía implícita entre las diversas fórmulas (la más "económica" para las familias es la ayuda en casa), sino que, por mucho que se presente como "excepcional", se está promoviendo indirectamente esta opción. La carencia de recursos que caracteriza a gran parte de familias podría incluso promover el efecto perverso de que, más que replantear las asunciones implícitas en una construcción sexuada de los cuidados, se esté favoreciendo la retirada de algunas mujeres del mercado de trabajo o la reducción de su jornada laboral para poderse hacer cargo de sus familiares dependientes (Abellán y Esparza 2010). El pago que se realiza al cuidador personal permite valorizar parcialmente estas actividades de cuidados, pero dada la precariedad de la situación de gran número de mujeres en el mercado de trabajo, la posible, aunque escasa, remuneración de estas actividades podría disuadirles de participar en el mercado de trabajo, contribuyendo a feminizar, aún más, el ejercicio de estas tareas.

En este sentido, se produce un doble proceso contradictorio. Por un lado, la retribución económica para el ejercicio de los cuidados permite visibilizar y valorizar estas actividades. Por otro, la desvalorización bajo la que se plantea esta opción cuando se vincula con un referencial femenino (familiar, informal, moral), contribuye a afirmar un imaginario que refuerza la asimetría entre los sexos. Un ejemplo que expresa esta situación es la ambigüedad en la definición del estatus de cuidadora, al situarse en un limbo jurídico entre lo familiar y lo mercantil ${ }^{11}$. Por tanto, esta producción de estatus híbridos

\footnotetext{
${ }^{11}$ Se asigna una prestación para los cuidados en el entorno familiar, sin embargo esta prestación no es directa, ni en forma de salario, sino que se concede a la persona en situación de dependencia. Por otro lado, aunque se regula la Seguridad Social de las personas cuidadoras en esta situación (Real Decreto 615/2007), sin embargo, su estatus no es de trabajador, sino que está reglamentado a través de un convenio especial y,
} 
entre lo familiar y lo mercantil no necesariamente replantea la dicotomía entre cuidados y trabajo productivo, y, por tanto, difícilmente va a poderse revisar la jerarquía de valores atribuidos socialmente a estos dos tipos de actividades.

Es más, la fórmula adoptada de otorgar una compensación económica sin cuestionarse el valor de estas actividades explica que se responda en muchos casos a las demandas contradictorias de la sociedad a través de una descentralización de los cuidados hacia colectivos con un menor estatus social, esto es, mujeres inmigrantes. Es más, el estatus jurídico que regula la Seguridad Social de las personas cuidadoras en el hogar plantea incompatibilidades con la actual Ley de extranjería, lo que deriva en la imposibilidad de las mujeres migrantes para renovar sus permisos de residencia y, por tanto, para la normalización de su estatus de ciudadanía en el país. De este modo, en esta cadena de los cuidados se van configurando nuevas jerarquías. A las divisiones tradicionales establecidas entre mujeres y hombres, se unen las constituidas en el seno mismo de las mujeres, en función de su distancia al espacio simbólico propio de lo masculino (público, profesional, certificado, racional, etc.).

\section{Representación de los cuidados}

Una visión despolitizada de la dependencia y familiarista del sujeto de los cuidados, que impugnan diversos grupos sociales, tiene también consecuencias en el modo de conceptualizar la naturaleza de la prestación que los servicios públicos deberían dirigir a las personas en situación de dependencia. La ley no desarrolla específicamente en qué términos se va a llevar a cabo el ejercicio de los cuidados ${ }^{12}$, pero sí plantea dos principios nucleares que han inspirado desarrollos legislativos posteriores, la profesionalización y la universalización de los cuidados, tal vez por existir un notable consenso entre los diversos actores sociales en que estas dos dimensiones son clave en la institucionalización de los cuidados.

Uno de los desarrollos legislativos más importantes en la ley ha sido la formalización de los criterios de profesionalización de los cuidados (Real Decreto 1224/2009). Un discurso que orienta la mirada al control de la calidad del servicio que se ofrece al usuario es unánimemente aceptado, aunque por razones muy diferentes, por los diversos actores sociales. Las asociaciones patronales, junto con los representantes de organizaciones de la tercera edad, han insistido mucho en una concepción profesionalizada de los cuidados, ya que les permite desarrollar un nicho en el mercado, cuyo valor añadido se basaría en el establecimiento de claras distancias simbólicas con aquellas actividades realizadas en el espacio familiar.

por tanto, no tiene acceso a la negociación jurídica, ni derecho a prestación por desempleo, ni a prestaciones por maternidad, paternidad o incapacidad temporal (sólo tiene cobertura para la jubilación y para la incapacidad permanente, muerte y supervivencia).

${ }^{12}$ Aunque el título II desarrolla una serie de medidas para garantizar la calidad de estos servicios de atención y cuidados. 
"La ley es una ley de servicios profesionales, que prevé de manera excepcional siempre que no haya servicios profesionales, la figura de un cuidador familiar. ¿Qué ocurre? (...) que lo que era una excepción se ha convertido en lo que está optando la mayoría. Porque además esta es una ley, donde al contrario de la Ley de sanidad o de educación donde es anticonstitucional que tú eduques a tus hijos en casa, aquí prevalece la opinión del familiar o del dependiente en este caso, por encima de la del profesional. Es como si yo fuera a un hospital y me dicen, mira, te tienes que operar de apendicitis, o tienes el brazo roto, y le digo, no me lo arregle usted, usted me da 400 euros, que ya me pongo yo unas vendas, me lo atiendo en casa. $Y$ eso es lo que está pasando con esta ley" (Representante de AESTE).

Esta orientación plantea una concepción del cuidado cuya acepción semántica dependería de su espacio de ejercicio. Induce una visión que valoriza aquellas actividades de cuidados ubicadas semánticamente en un espacio vinculado con lo masculino (remunerado, certificado y regulado). Esta visión que, por un lado permitiría facilitar la desfamiliarización de los cuidados, tiene también el efecto perverso de reproducir las valoraciones dicotómicas en las que se ha basado el orden social del género, induciéndose la formación de nuevas jerarquías según la distancia de la mujer o varón a la norma de masculinidad. Las voces de movimientos de mujeres también apoyan este marco de profesionalización, pero, en este caso, por oposición a una concepción familiarista, y defienden una profesionalización de los cuidados garantizada por los poderes públicos.

"Tanto dentro del movimiento como en todo ese entorno, que está gestionando la ley, sabe que las feministas estamos en contra de perpetuar los cuidados en el seno de la familia y que pedimos servicios profesionales y públicos, no queremos pagas ni 'paguitas', queremos servicios" (Representante del Forum de Política Feminista).

Tras esta apelación a la profesionalización se están activando importantes ritos de institución, que asignan esencias y propiedades a determinados tipo de actividades. Esta noción de profesionalización se muestra vinculada a nociones como instrucción, formación, calidad, etc. en donde aparece implícito un concepto de sujeto, como es el propio del discurso educativo, y cuyas características vendrían definidas por su carácter incompleto, por aquello que todavía no es, por su articulación en base a estatus o niveles que el sujeto, de forma inexorable, tendría que recorrer (y mostrar a través de un certificado) para llegar al estadio final (el profesional). Este estatus que cualifica la actividad vendrá acreditado por unos rituales que certifican que el proceso se ha desarrollado de la forma esperada. Estos certificados permiten consagrar las diferencias e instaurar un límite y división entre los cuidados profesionales y no profesionales.

Este proceso de evaluación y acreditación de las competencias, desarrollado en el Real Decreto 1224/2009, permite, por un lado, visibilizar (describir, analizar y racionalizar) la compleja movilización de competencias tácitas, intangibles, informales, sumamente complejas (Durán 2002; Torns 2007), que reclaman el desarrollo de este tipo de 
tareas; pero, por otro lado, contribuye a jerarquizar las actividades humanas y a reproducir dicotomías asimétricas entre las actividades domésticas, no profesionales, descualificadas y gobernadas por imperativos morales, y las actividades laborales, profesionales, cualificadas y gobernadas por procedimientos técnicos, y confirmadas por un protocolo institucional como es el sistema de acreditación de cualificaciones. De este modo, por un lado, la profesionalización de los cuidados plantea un importante potencial emancipador ya que induce una "mirada" en una dirección opuesta a la anterior (de una invisibilización de los cuidados a un registro y codificación sistemática de las competencias vinculadas a los cuidados), pudiéndose promover así el ennoblecimiento de este tipo de actividades. Sin embargo, esto se desarrolla no por dignificarse el "sexo" de los cuidados sino porque este tipo de actividades es sometido a un proceso de "purificación" ritual en el espacio social atribuido a lo masculino (curiosamente, el proceso contrario no ha tenido lugar: esto es, la colonización en el espacio laboral mercantil de lógicas atribuidas a la ética de los cuidados, como son la producción del vivir, la liberación de la búsqueda obsesiva de rendimiento, la solidaridad con el sujeto de necesidad, etc.).

Junto a esta profesionalización de los cuidados, se está induciendo la mercantilización de la prestación, que convierte al dependiente en cliente soberano. La cuestión de la libertad de elección ha sido una demanda ampliamente defendida tanto por movimientos de la tercera edad como de diversidad funcional (Rodríguez Cabrero 2011). Una concepción individualizadora orienta gran parte de la discusión hacia la identificación de los mecanismos que permitan satisfacer las necesidades de un cliente, receptor de estos servicios, $y$ en donde las infraestructuras sociales ofrecidas por los poderes públicos serían así comparadas a las infraestructuras hoteleras.

"Una residencia, por ejemplo, una persona que vive en una residencia no tiene gastos (...) Aquí no tiene gastos, no tiene que pagar alojamiento, tiene unos servicios..., de lavandería. Tiene una limpieza. Tiene comida. Tiene una serie de servicios que llamamos pues que son..., hoteleros. Y tiene unos servicios asistenciales (...) No podemos obligar a la gente. Esto no es una pena. Es decir: 'Usted, condenado. Tiene dependencia, además, está inhabilitado porque tiene Alzheimer; y yo decido que lo mejor para usted es tal sitio"'. Pues no podemos entrar en eso (Representante 3 del IMSERSO).

Esta noción individualizadora de lo que significa el cuidado puede inducir una discusión del problema de la dependencia en términos mercantilistas (Rodríguez Cabrero 2011), según la cual, la autonomía significa la libertad de un cliente para decidir aquellos servicios que se consideren más adecuados a su situación personal. El debate omitido en esta concepción médico individualista de lo que es la dependencia es qué modelo de protección social estamos fomentando, quién es responsable de qué, y en qué medida este modelo está capacitando el empoderamiento político de ambos, personas atendidas y sujetos de los cuidados, objetivo este invocado en la ley.

En esta cuestión, las voces representativas de los movimientos de mujeres consideran que un exclusivo énfasis en la soberanía del dependiente, que apoya esta visión 
mercantilizadora de los servicios ofrecidos, omite cualquier análisis político en donde se plantee una reflexión global acerca de la necesidad de cambiar el modelo familiarista y privatizado que se impone con la opción, aunque se presente en la ley como extraordinaria, de remunerar mínimamente al cuidador familiar.

"Nosotros lo que pedimos es que todos sean servicios profesionales, (...) es decir, primero el que no haya prestaciones económicas ni cheques servicios, y que todos sean servicios, sean servicios profesionales, nada de servicios familiares y que sean públicos" (Representante del Forum de Política Feminista).

Por tanto, aunque muchos actores (técnicos del IMSERSO, representantes de organizaciones de mayores, movimientos de mujeres, actores sindicales, patronal, etc.) coinciden en el discurso de la profesionalización de los cuidados; sin embargo, el significado de esta noción de profesionalización es extremadamente polisémico. No necesariamente implica una revisión del espacio "natural" para el ejercicio de los cuidados, sino que se reduce, en muchas ocasiones, a reclamar una mejora en la calidad de este servicio. En este caso, la demanda de profesionalización viene promovida, no tanto (o no siempre) por un cuestionamiento de la moralidad o justicia de la familiarización de los cuidados, sino por una sospecha acerca de la calidad de estas prestaciones.

"Si queremos y decimos que queremos servicios profesionales frente a los cuidados familiares, para que sean servicios profesionales tienen que tener formación, tienen que tener cualificación. 0 sea, no puede ser cualquiera el que valga para atender a las personas en situación de dependencia" (Representante del IMSERSO).

Puede tener además el efecto perverso de inducir la invasión panóptica de las antenas del estado en espacios hasta ahora ajenos a la presencia estatal, como son el seno del hogar ${ }^{13}$.

Otra de las cuestiones en donde predomina un gran consenso es la cuestión de la universalización de esta prestación, que ha sido una de las principales señas de identidad de la ley. No hay, sin embargo, tal acuerdo en identificar cuál es el modelo de financiación (financiación impositiva, basado en la seguridad social o asistencial) de esta prestación, ya que, si algunos actores, como los representantes de los trabajadores han respaldado la opción basada en la Seguridad Social, la patronal, apoyada por las Comunidades Autónomas, defendían un modelo impositivo.

Aunque todos los actores sociales que están pugnando por participar en el proceso de construcción de la ley destacan la importancia de asegurar el derecho político de

\footnotetext{
${ }^{13}$ En virtud del Acuerdo del Consejo Territorial del 4 de febrero de 2010, de la Secretaría General de Política Social y Consumo, los servicios sociales deberán certificar que se cumplen en el hogar las adecuadas condiciones de convivencia y relación.
} 
carácter universal a recibir asistencia, unos actores inciden en el papel que puede jugar el mercado, otros abanderan la defensa de un sistema público que entienden como garantía de esta universalidad y de responsabilización colectiva. Y a pesar de esta mezcla híbrida de marcos, no es lo mismo plantear esta intervención apelando a un marco público de justicia redistributiva que hacerlo en términos de rentabilidad económica. Esta situación favorece que este cuarto pilar del estado social sea concebido también como espacio para la creación de nuevos yacimientos de empleo y de modos de promover la economía social. Sin embargo, la prioridad que se otorga en la práctica a las prestaciones económicas (aunque en la ley se consideren excepcionales), la escasez de recursos económicos (el sistema queda relegado a aquellas dependencias más severas), los límites que plantea la noción de dependencia (la dependencia tiene que ser permanente, y vinculada a disfuncionamientos físicos), la responsabilización de los beneficiarios a través del copago (que implica la participación del dependiente una vez considerada su renta y patrimonio: el $33 \%$ del coste del servicio de media), las diferencias en el acceso en función de la Comunidad Autónoma que corresponda, o las diferencias en el acceso entre migrantes y autóctonos, etc. cuestionan el carácter universal y público de esta ley.

\section{CONCLUSIONES}

El punto de partida de esta reflexión destacaba el importante potencial transformador de la LAAD al poder inducir, aunque sea de forma indirecta, el cuestionamiento de gran número de a prioris que han hecho realidad la regulación moral de los cuidados. Sin embargo, el análisis de la forma concreta que ha adoptado la ley desde una perspectiva de género manifiesta el tímido ejercicio emancipador y desestabilizador de los imaginarios políticos en los que se ha construido el orden social de género. Por tanto, intervenciones frente a la crisis de los cuidados como la que persigue la LAAD son esenciales en términos de justicia social. Sin embargo, es fundamental que estas intervenciones incorporen una concepción mucho más amplia de lo que significa la vulnerabilidad.

El análisis de la LAAD pone de manifiesto la naturaleza ambigua, polifónica, y polisémica de nociones como los cuidados, la dependencia, la profesionalización, etc., lo que denota cómo es el signo un espacio esencial en donde se lidian gran número de batallas entre grupos sociales que pugnan por el sentido. El signo, como planteó Bajtin (1986), es semánticamente indeterminado e inconcluso, ya que representa la materialización de un conflicto entre grupos sociales. En el seno de cada concepto se aloja una diversidad de voces que no son sino expresión de una multiplicidad de puntos de vista. El carácter multivocal de textos como el documento de la LAAD no evita que haya unas voces que aparezcan como hegemónicas a lo largo del texto. El particular procedimiento de realización del proyecto de ley junto a una serie de condiciones contextuales, como son la insuficiencia financiera del sistema, el margen de maniobra del que disponían diversos 
grupos sociales frente a la ley, el modelo cultural que sustenta el orden político entre los sexos, los bajos niveles de oferta de servicios sociales, la fragilidad asociativa y laboral de las cuidadoras, la estructura político institucional del país, explican la particular visión que la ley plantea sobre los cuidados.

La hegemonía de una visión gerontológica de la dependencia, más que facilitar la revalorización y reconocimiento de este mundo desconocido del cuidar, ha acentuado una valoración dicotómica de los espacios políticos del cuidar. A esto se ha unido una representación mercantilizante de la prestación que restringe el derecho a la promoción de autonomía, al desarrollo de las condiciones de ejercicio de la soberanía de un cliente que puede acceder libremente a los servicios. El énfasis en la profesionalización y desfamiliarización ha sido el resultado de una concepción de la autonomía vinculada a la soberanía del cliente, y en donde se asume sólo el valor de aquellas actividades marcadas por los rituales de autoridad característicos del espacio masculino (certificación profesional, carácter público, ámbito extra-familiar, etc.). De este modo, estas políticas, más que reducir la feminización de los cuidados, la reconstruye y adapta (Asamblea feminista 2006), usando nuevas tecnologías de regulación, como son el contrato. Esta situación no facilita la impugnación de las asunciones que mantienen el orden social de género, sino que, más bien, estimula un proceso en donde las jerarquías no se renuevan sino que se multiplican según su distancia a la norma de masculinidad sin cuestionar su orden esencial.

La acepción de vulnerabilidad presente en esta ley es muy distinta a aquella que inspiró la legislación protectora más potente en las sociedades occidentales, la protección social laboral, en donde la vulnerabilidad ha sido vinculada a las relaciones asimétricas de fuerzas o relaciones de explotación que dificultan la capacidad de acción de los sujetos. La lectura que se hace de la vulnerabilidad no es política, sino individualizante, aunque no responsabilizante. Si la conceptualización del problema de la vulnerabilidad vinculada con el trabajo activó marcos que establecían una conexión directa con relaciones de poder y opresión, en el caso de la vulnerabilidad vinculada con la edad, se establecen más bien marcos reparadores y terapeutizantes, que posibiliten al sujeto recuperar su autonomía. Esta representación hegemónica de la dependencia y de los cuidados promueve la despolitización e individualización de los cuidados al desconsiderarse el contexto político y social que regula el ejercicio de la actividad. Esta concepción despolitizada de la dependencia omite cualquier reflexión acerca de la distribución naturalizada de las responsabilidades en el ejercicio de los cuidados, del significado asignado al hecho de cuidar y a la condición de dependencia. En este sentido, el modelo que se desarrolla en España es diferente al abordado en otros países europeos como los nórdicos, en donde el sistema se constituyó en parte como respuesta a las demandas de las mujeres trabajadoras (Rodríguez Cabrero 2011) ${ }^{14}$. Una posible línea de investiga-

\footnotetext{
${ }^{14}$ Frente al modelo de institucionalización de los cuidados de los países nórdicos, caracterizado por proporcionar una cobertura universal fundamentalmente pública, en los países mediterráneos, dentro de los cuales está España, persisten modelos de provisión de los cuidados fundamentalmente familiaristas (Rodrí-
} 
ción futura, que reclama una perspectiva comparativa transcultural, sería el análisis de la contribución de movimientos sociales de diversos países, particularmente movimientos de mujeres, al diseño semántico y político de la ley, y su posible efecto en la acentuación del potencial transgresor de esta, como ocurrió en el caso de la participación de los movimientos sociales de trabajadores en la producción y regulación del trabajo a finales del s. XIX y a lo largo del siglo XX.

Por tanto, la formulación de esta ley, lejos de replantear y someter a disputa gran parte de las concepciones subyacentes en el orden político del género, contribuye a reproducirlas, estableciéndose categorías y divisiones dentro de las mismas mujeres según la cercanía de estas al espacio social atribuido a lo femenino (informal, emocional y doméstico). La escasez de recursos que se han movilizado para la aplicación de la ley, escasez esta que se ha visto acentuada tras la crisis económica, la configuración institucional del poder entre los diversos niveles geográficos, la carencia de infraestructuras públicas de atención a la dependencia, etc. no hacen sino acentuar estos riesgos identificados en la redacción de la ley.

La institucionalización y politización del riesgo de dependencia no ha planteado su transformación, sino la movilización de los recursos existentes para adaptarlos a las nuevas condiciones, reforzándose la responsabilidad familiar. A las obligaciones vinculadas a vínculos morales, se suman las propias de una relación mercantil, fortaleciendo su familiarización. Además, parte del riesgo es trasladado a la persona dependiente (el individuo asume mayor responsabilidad bajo el pretexto de ofrecer un servicio de mayor calidad), y con la movilización del sector mercantil (desgravaciones fiscales), se refuerza la idea de que la dependencia es un riesgo privado (Rodríguez Cabrero 2011). La representación de la dependencia que induce esta ley omite el hecho de que lo contrario de la dependencia no es necesariamente la autonomía; que la dependencia es una condición inmanente a todo sujeto y que son las condiciones de vulnerabilidad, no sólo material sino también política, lo que hace a la dependencia una condición susceptible de ser combatida. Junto al papel central de los modelos culturales imperantes en cada país y las inercias institucionales, la escasa presencia de las voces y perspectivas de los movimientos de mujeres son factores importantes para explicar la ausencia de una representación que permita visibilizar las relaciones políticas que se asumen en el ejercicio de los cuidados.

guez Cabrero 2011; Esping-Andersen 1990). Sin embargo, hay también que destacar que se está produciendo una cierta convergencia en el seno de la UE en la institucionalización de los cuidados, como resultado del nuevo equilibrio político de fuerzas, que se está traduciendo en una mayor prioridad otorgada al ámbito mercantil, y está favoreciendo nuevos desplazamientos hacia la esfera familiar y a la feminización de los cuidados (Arriba y Moreno 2009; Rodríguez Cabrero 2011). Esta situación induce una tendencia común hacia la preferencia por las prestaciones monetarias frente a los servicios sociales, la promoción de la libertad de elección, la mercantilización de la provisión, el copago, el establecimiento de un baremo para la dependencia que prioriza las situaciones más graves, junto a otros mecanismos que promueven la individualización del riesgo social de la dependencia. 
La existencia de iniciativas políticas como la $L A A D$, sin cuestionarse otras condiciones sociales que reproducen la asimetría de poder, como son el peso que juega la cultura moral con la que se regulan las relaciones familiares, la ausencia de servicios sociales y otras infraestructuras para responder a la demanda de cuidados, la escasa presencia de opciones institucionales y estrategias educativas para que los varones puedan optar por cuidar, la precariedad de un mercado de trabajo que perjudica de forma notoria a los más frágiles, las posiciones de poder de distintos grupos sociales, etc. impiden, en gran medida, el replanteamiento de los axiomas indiscutibles bajo los que se sustenta la división y jerarquización social y sexual de las actividades sociales.

\section{REFERENCIAS BIBLIOGRÁFICAS}

Artiaga Leiras, A. y Serrano Pascual, A. 2012. "Los cuidados y la Ley de dependencia: pugnas políticas y nuevas divisiones del orden social", en Libro de actas del Primer Congreso "Trabajo, Economía y Sociedad". Alternativas para una salida social a la crisis. 21/22 de junio de 2012. Fundación $1^{0}$ de Mayo.(http://www.1mayo.ccoo.es/nova/NBdd_ShwDocumento?cod_primaria=1185\&cod_documento=4009)

Abellán, A. y Esparza, C. 2010. "Solidaridad familiar y dependencia entre las personas mayores." Informes Portal Mayores 99. Consulta 15 de Junio de 2012 (http://www.imsersomayores.csic.es/ documentos/documentos/pm-solidaridad-familiar.pdf).

Arriba, A y Moreno, J. comp. 2009. El tratamiento de la dependencia en los regímenes de bienestar europeos contemporáneos. Madrid: IMSERSO.

Asamblea Feminista. 2006. "La Ley de dependencia ante la crisis del trabajo de cuidados." Cuaderno Feminista 2. Consulta 15 de Junio de 2011 (http://www.nodo50.org/feministas/madrid).

Bajtin, M. [1979] 1986. Problemas de la poética de Dostoievski. México: FCE.

Bettio, F., Simonazzi, A., y Villa, P. 2006. "Change in care regimes and female migration: the care drain in the Mediterranean." Journal of European Social Policy 16(3): 271-285.

Bourdieu, P. [1985] 2001. ¿Qué significa hablar? Economía de los intercambios lingüísticos. Madrid: Akal.

Bustelo, M. y Lombardo, E. (Eds.) 2007. Las políticas de igualdad en España y en Europa. Madrid: Editorial Cátedra.

Casado, D. comp. 2004. Propuestas a la dependencia. Madrid: CCS.

Carrasco, C., Borderías, C. y Torns, T. comp. 2011. El trabajo de cuidados. Historia, teoría y políticas. Madrid: Los Libros de la Catarata.

Carrasquer, P. 2013. "El redescubrimiento del trabajo de cuidados: algunas reflexiones desde la sociología." Cuadernos de Relaciones Laborales (en prensa).

Daly, M. y Lewis, J. 2000. "The concept of social care and the analysis of contemporary welfare states." British Journal of Sociology 51(2):281-298. 
De Villota, P. Comp. 2008. Conciliación de la vida profesional y familiar: políticas públicas de conciliación en la Unión Europea. Madrid: Síntesis.

Díaz Velázquez, E. 2010. “¿Políticas sociales de dependencia o de promoción de la autonomía?” Zerbitzuan 48:33-43.

Dizy, D. 2010. Dependencia y familia: una perspectiva socio-económica. Madrid: IMSERSO. Consulta 15 de Junio de 2011 (http://www.imsersomayores.csic.es/documentos/documentos/dizy-dependencia-01. pdf).

Durán, M. A. 2002. Los costes invisibles de la enfermedad. $2^{a}$ ed. Bilbao: Fundación BBVA.

Esping-Andersen, G. 1990. The Three Worlds of Welfare Capitalism. Londres: Polity Press.

Fairclough, N. 1995. Critical Discourse Analysis. Londres: Longman.

Fraser, N. 1989. "Women, welfare and the politics of need interpretation." Pp. 145-160 en Unruly Practices: Power, Discourse and Gender in Contemporary Social Theory. Cambridge: Policy Press.

García Ruíz, A. 2011. "En extraña compañía: la tercera persona y las filosofías de lo impersonal." Pp.71-104 en Las personas del verbo, editado por M. Cruz. Barcelona: Herder Editorial.

Guzmán, F., Moscoso, M. y Toboso, M. 2010. "Por qué la ley de Dependencia no constituye un instrumento para la promoción de la autonomía personal." Zerbitzuan 48:43-55.

Herce, J.A., Labeaga, J.M., Sosvilla, S., y Ortega, C. 2006. Sistema Nacional de Dependencia. Evaluación de sus efectos sobre el empleo. Madrid: IMSERSO.

Hirata, E. y Zariffia, P. 2007. "El concepto de trabajo." Revista de Trabajo 4:33-36.

Illouz, E. 2010. La salvación del alma moderna. Madrid: Katz editores.

Instituto Regional de las Cualificaciones de la Comunidad de Madrid. 2010. Estudio de los perfiles existentes y las necesidades formativas en el ámbito de las actividades ligadas a la ley de depen-dencia. Consulta 10 de Junio de 2012 (http://www.asociacion-aeste.es/comun/documentacion/ircual.pdf).

Lewis, J. 2011. "Género, envejecimiento y el nuevo pacto social: la importancia de desarrollar un enfoque holístico de las políticas de cuidados." Pp. 336-359 en El trabajo de cuidados. Historia, teoría y políticas, editado por C. Carrasco, C. Borderías y T. Torns. Madrid: Los Libros de la Catarata.

Marbán, V. 2012. "Actores sociales y desarrollo de la Ley de Dependencia en España." Revista Internacional de Sociología 70(2):375-398.

Martín Palomo, T. 2010. "Autonomía, dependencia y vulnerabilidad en la construcción de la ciudadanía." Zerbitzuan 48:57-69.

Montserrat, J. 2006. "La Ley de dependencia: costes y financiación." Documentación social 141:65-83.

Nancy, J. L. 2006. El intruso. Buenos Aires: Amorrortu.

Observatorio de la Discapacidad. 2008. "Publicados los primeros resultados sobre nueva encuesta de discapacidad "EDAD 2008". Minusval Noviembre/Diciembre: 38-39. Consulta 21 de Mayo de 2012 (http://www.bsocial.gva.es/accesible/portal?docid=6887).

Observatorio de Personas Mayores. 2008. "Cuidadoras y cuidadores: el efecto del género en el cuidado no profesional de los mayores." Boletín sobre el envejecimiento: Perfiles y Tendencias 35. 
O'Connor, J. 1996 "From Women in the Welfare State to Gendering Welfare Regimes." Current Sociology 44(2):1-130.

Orloff, A. S. 1993. "Gender and the social rights of citizenship: state policies and gender relations in comparative research." American Sociological Review 58(3):303-328.

Pérez, A. 2006. Perspectivas feministas en torno a la economía: el caso de los cuidados. Madrid: CES.

Peterson, E. y Bustelo, M. 2007. "Care Politics and invisible workers. The emergence of care and domestic work issues in Spanish policy debates." Conferencia presentada en 4th ECPR General Conference. 6-8 Septiembre, Pisa, Italia.

Rodríguez Cabrero, G. 2007. "La protección social de la dependencia en España. Un modelo sui generis de desarrollo de los derechos sociales." Política y Sociedad 44: 69-85.

Rodríguez Cabrero, G. 2010. "El desarrollo de la política social de promoción de la autonomía y atención a las personas en situación de dependencia en España (2007-2009)." Gestión y Análisis de Políticas Públicas 3:33-54.

Rodríguez Cabrero, G. 2011. "Políticas sociales de atención a la dependencia en los Regímenes de Bienestar de la Unión Europea." Cuadernos de Relaciones Laborales 29:13-42.

Rogero, J. 2010. "Los tiempos del cuidado. El impacto de la dependencia de los mayores en la vida cotidiana de sus cuidadores." Tesis doctoral, Departamento de Sociología I (Cambio Social), Universidad Complutense de Madrid.

SAAD-IMSERSO. 2012. Estadísticas del sistema para la autonomía y la Atención a la dependencia. Consulta 15 de Noviembre de 2012 (http://www.dependencia.imserso.es/InterPresent2/groups/ imserso/documents/binario/im_063871.xls).

SIPOSO. 2008. Anotaciones a la LAAD. Madrid: Polibea. Londres.

Sainsbury, D. ed. 1994. Gendering Welfare States. Londres: Sage.

Torns, T. 2007. "El cuidado de la dependencia. Un trabajo de cuidado." Revista Mientrastanto 103: 33-43.

Turner, V. 1997. The forest of symbols: aspects of Ndembu Ritual. Ithaca: Cornell University Press.

Weiss, G. y Wodak, R. 2008. Critical Discourse Analysis: Theory and Interdisciplinarity. Londres: Palgrave Macmillan.

Amparo Serrano Pascual es Profesora de la Facultad de Ciencias Políticas y Sociología de la Universidad Complutense de Madrid. Autora de numerosos trabajos en diversos países europeos (España, los Países Bajos, Italia, Francia, Bélgica, Reino Unido, etc.) y fuera de la UE (EEUU, Japón), siendo el libro más reciente: Serrano, A. (con M. Keune, coord.) (en prensa) Alternatives to flexicurity, New York: Routledge.

Alba Artiaga LeiRas es Investigadora de la Universidad Complutense de Madrid. Principales líneas de investigación: políticas públicas, género y trabajo, los cuidados, regulación social del trabajo. Entre sus últimas publicaciones se encuentra Ingenierías de la subjetividad: el caso de la orientación para el empleo (REIS, Madrid, 2012; junto con A. Serrano Pascual y C. J. Fernández Rodríguez). 
María Celeste Dávila de León es Profesora de la Universidad Complutense de Madrid. Principales líneas de investigación: conducta prosocial, voluntariado y comportamiento de ciudadanía organizacional. Una de sus publicaciones más recientes es Dávila, M. C (con Finkelstein, M.A. y Castien, J.I). (2010). Diferencias de género en conducta prosocial: el comportamiento de ciudadanía organizacional. Anales de Psicología, 27(2),498-506.

RECIBIDO: $30 / 07 / 2012$

ACEPTADO: 27/11/2012

Publicado on-line: 16/05/2013 